PROBLEM-SOLVING TRAINING

RUNNING HEAD: PROBLEM-SOLVING TRAINING

Draft version 2.0, 14 December 2020 - This paper has not been peer reviewed.

\title{
Problem-Solving Training as an Active Ingredient of Treatment for Youth Depression: A Scoping Review and Meta-Analysis
}

Karolin R. Krause ${ }^{1,2}$, Darren B. Courtney ${ }^{1,3}$, Benjamin W. C. Chan ${ }^{4}$, Sarah Bonato ${ }^{1}$, Madison Aitken ${ }^{1,3}$, Jacqueline Relihan ${ }^{1}$, Matthew Prebeg ${ }^{1}$, Karleigh Darnay ${ }^{1}$, Lisa D. Hawke ${ }^{1,3}$, Priya Watson ${ }^{1,3}$, Peter Szatmari ${ }^{1,3,5}$

1) Cundill Centre for Child and Youth Depression, Centre for Addiction and Mental Health (CAMH), Toronto, ON, Canada

2) Evidence-Based Practice Unit, University College London and Anna Freud National Centre for Children and Families and, London, United Kingdom

3) Department of Psychiatry, University of Toronto, Toronto, ON, Canada

4) Independent Family Doctor, Toronto, ON, Canada

5) Hospital for Sick Children, Toronto, ON, Canada

Corresponding Author: Karolin Krause, Cundill Centre for Child and Youth Depression, Centre for Addiction and Mental Health, 80 Workman Way, Toronto, ON M6J 1H4, Canada; Email: Karolin.krause@ camh.ca

Disclosure of conflict of interest: The authors have no conflicts of interest to declare.

Funding: This work was funded by a Wellcome Trust Mental Health Priority Area 'Active Ingredients' commission awarded to Dr Peter Szatmari and the Centre for Addiction and Mental Health, Toronto, Canada.

Acknowledgments: We would like to thank the members of the Centre for Addiction and Mental Health (CAMH) youth advisory group for their valuable insights and suggestions. The systematic search for Clinical Practice Guidelines presented in this view was based on a search strategy developed by Dr Kathryn Bennett. We would like to thank Dr Bennett for agreeing to the reuse of this strategy as part of this review. We would also like to thank the Cundill Centre for Child and Youth Depression for providing institutional support to this project.

Data availability: The authors confirm that the data supporting the findings of this study are available within the article and its supplementary materials.

Word Count: 5,972 words. 


\begin{abstract}
Background: Problem-solving (PS) training is a common ingredient of evidence-based therapies for youth depression that can be delivered as part of broader therapy packages or alone. As a stand-alone treatment, ProblemSolving Therapy (PST) is effective for adult depression. Aims: This scoping review aimed to provide an overview of the evidence concerning PS training for depression in youth aged 14 to 24. Method: We searched five bibliographic databases and the grey literature. We included four randomized control trials (524 participants) comparing PST with waitlist controls or comparator interventions; four secondary analyses of PS-related concepts as predictors, moderators, or mediators of treatment response; 23 clinical practice guidelines (CPGs); and insights from a youth advisory panel. Results: One high-quality study found PST helped with personal problems but was not significantly more effective than the control at reducing emotional symptoms. Exploratory evidence suggests PS training may enhance treatment effectiveness if provided alongside other skills in cognitive behavioural therapy. CPGs do not recommend PST as a first-line treatment for youth depression. Exploratory meta-analysis showed a small effect (Hedges' g $=-0.34 ; 95 \%$ CI: -0.92 to 0.23 ) with high heterogeneity and a very low quality of the evidence. After removing one study at high risk of bias, effect size and heterogeneity were strongly reduced ( $\mathrm{g}=-0.08$; $95 \% \mathrm{CI}$ : -0.26 to 0.10$)$. Conclusions: High-quality trials of PST alone or with other therapies are needed. As per suggestions by youth advisors, PS training may need to be reworked to ensure it is youth-driven, strengths-based, comprehensive, and personalized.
\end{abstract}


PROBLEM-SOLVING TRAINING

\section{Introduction}

Depressive disorders are common in adolescence and a leading contributor to health-related disability worldwide (1). Several psychotherapies have proven modestly effective at reducing youth depression, including cognitivebehavioural therapy (CBT) and interpersonal therapy (IPT) (2,3). Room for improvement remains; for around half of youth, symptoms do not measurably improve after an average of 30 weeks of routine clinical care for depression or anxiety (4). One barrier to greater impact is a lack of understanding of which treatment ingredients are most critical $(5,6)$. Identifying the "active ingredients" that underpin effective approaches, and understanding when and for whom they are most effective is an important avenue for enhancing impact (6). Distilling interventions to their most effective ingredients while reducing redundant content may help make treatment more efficient; that is, reduce treatment length and cost, freeing up resources to expand service provision. Given that youth tend to drop out of treatment early (7), introducing the most effective ingredients at the start may also help improve outcomes.

One common ingredient in the treatment of youth depression is problem-solving (PS) training (8). Problem solving in real-life contexts (also called social problem solving) describes "the self-directed process by which individuals attempt to identify [...] adaptive coping solutions for problems, both acute and chronic, that they encounter in everyday living" (9). Chronic problems may relate to difficulties at school, work, within the family, or peer group (e.g., bullying). Singular events may include challenges arising from parental divorce or bereavement (10). Within a relational/problem-solving model of stress and well-being, mental health difficulties are viewed as the result of maladaptive coping behaviours that cannot adequately safeguard an individual's well-being against such stressors (11). Indeed, maladaptive PS is associated with depressive symptoms in adolescents and young adults (12-16). In turn, effective PS can protect youth well-being against stress imposed by challenging events or daily problems $(11,17)$.

Based on a conceptual model developed by D'Zurilla and colleagues $(11,18,19)$, effective PS requires a constructive and confident attitude towards problems (i.e., positive problem orientation), and the ability to approach problems rationally and systematically (i.e., rational PS style). In turn, defeatist or catastrophizing attitudes towards problems (i.e., a negative problem orientation), passively waiting for problems to resolve (i.e., avoidant style), or acting impulsively without thinking through possible consequences and alternative solutions (i.e., impulsive/careless style) are considered maladaptive $(9,10,19)$. This conceptual model is illustrated in Fig 1. 


\section{PROBLEM-SOLVING TRAINING}

\section{Fig 1. Key Concepts and Components of PST}

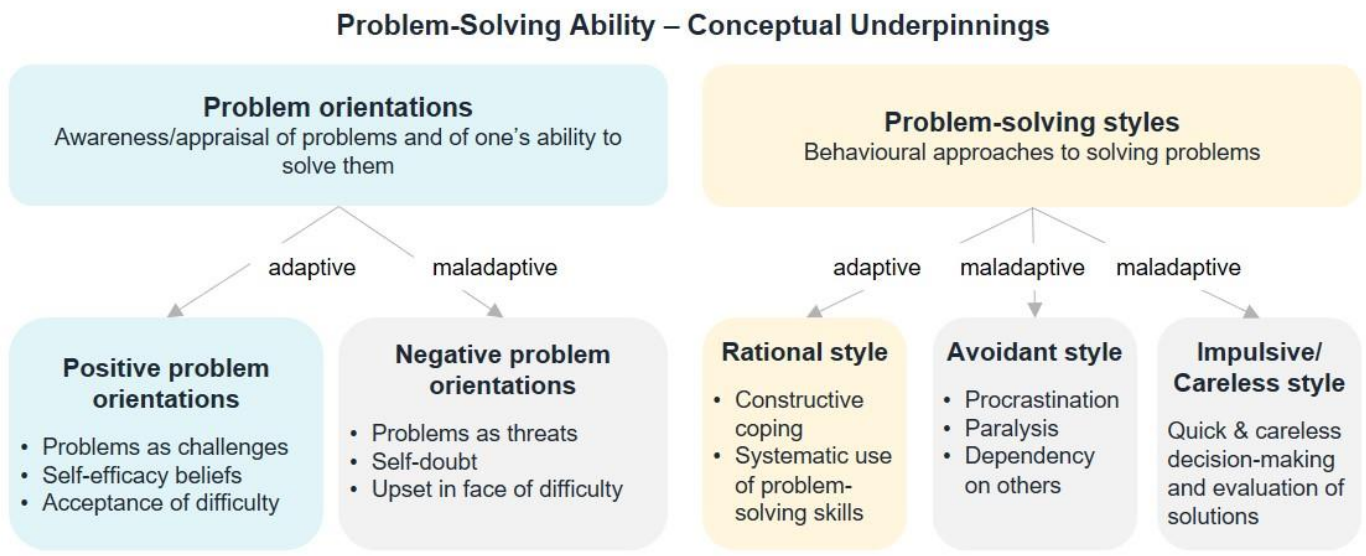

Aims of Problem-Solving Therapy

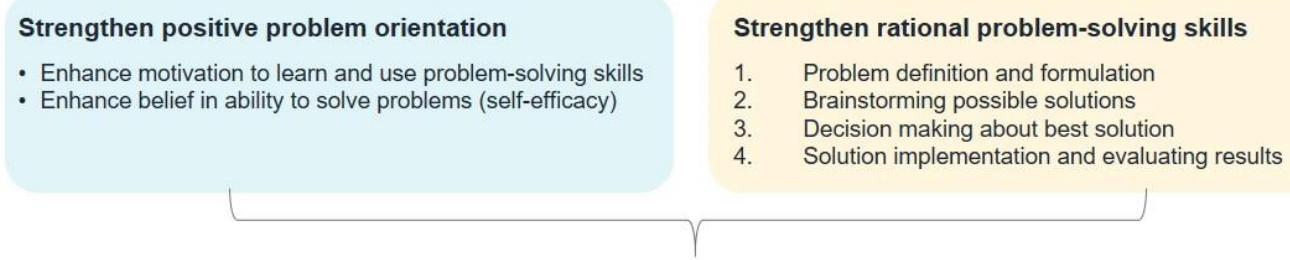

Modules of Problem-Solving Therapy (D'Zurilla \& Nezu 2010, p. 209) [11]

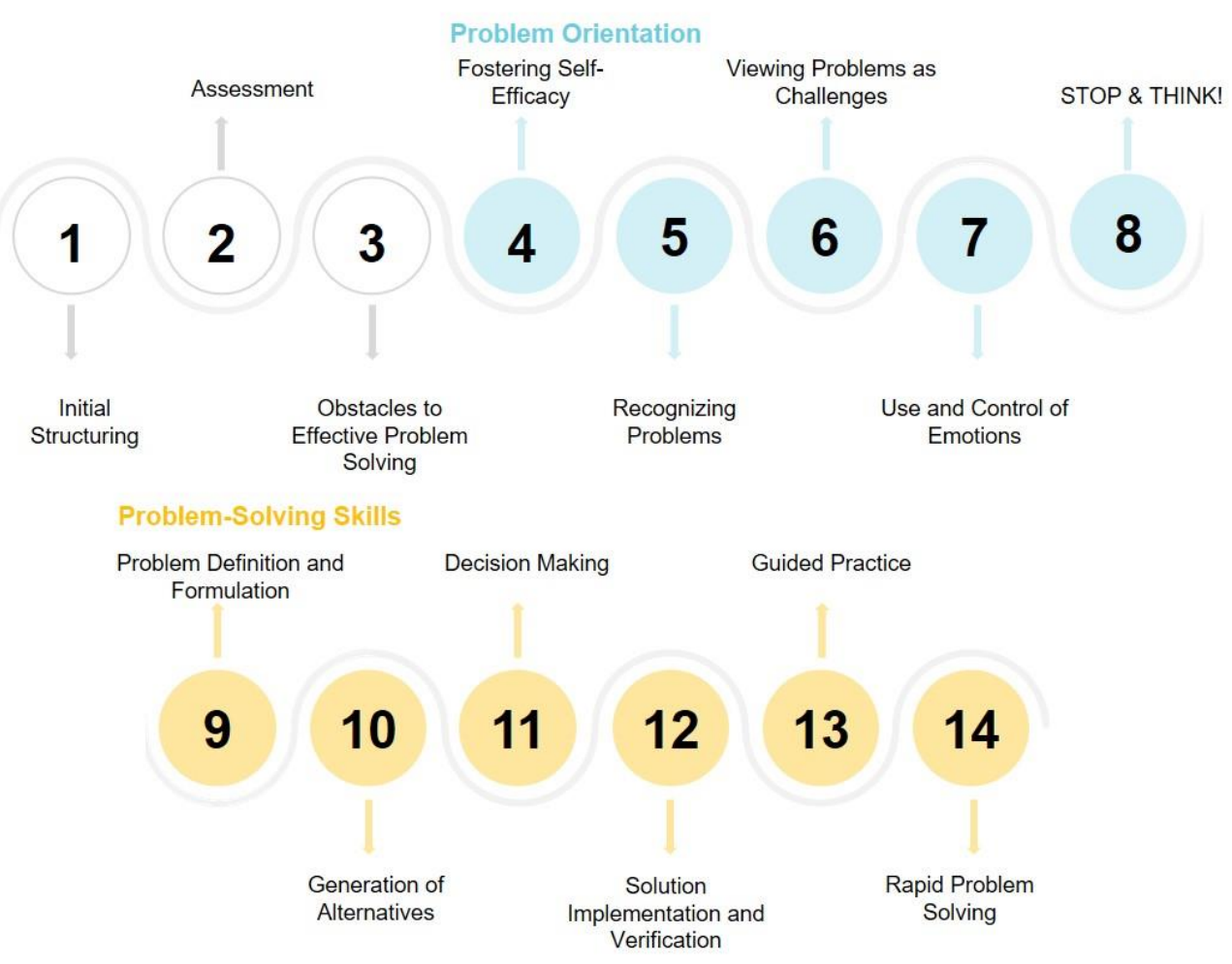

Note. This figure illustrates the concepts and components of PST as described by D'Zurilla \& Goldfried (1971) (19), Nezu et al. (2013) (9), and D’Zurilla \& Nezu (2010) (11). Topics of PST modules are presented verbatim as listed by D’Zurilla \& Nezu (2010, p. 209-210) (11). 


\section{PROBLEM-SOLVING TRAINING}

In the 1970s, D'Zurilla and Goldfried (20) introduced Problem-Solving Therapy (PST) as a therapeutic intervention rooted in Social Learning Theory (21). Meta-analyses considering over 30 randomized control trials (RCTs) of PST for adult depression suggest it is as effective as CBT and IPT and more effective than waitlist or attention controls (22-24). PST has been applied with children, adolescents, and young adults (25-30), although dedicated manuals for different developmental stages are not readily available. PST aims to foster a positive problem orientation, by teaching clients that challenges are an inherent feature of life and often can be overcome $(9,19)$. It further teaches a sequence of rational PS steps: (1) defining and formulating the problem; (2) brainstorming possible solutions; (3) appraising each solution and selecting the most promising one; (4) implementing the preferred solution and reflecting on the outcome. Clients are encouraged to internalize this sequence to better solve problems outside of therapy (10). The general content of PST is illustrated in Fig 1 (11). PST is distinct from Solution-Focused Brief Therapy (SFBT), which has different conceptual roots and emphasizes the construction of solutions over the in-depth formulation of problems (31).

PS training is also a common ingredient of other depression treatments $(8,10)$. PS skills are commonly taught within CBT and Dialectic Behaviour Therapy (DBT) $(8,32-35)$, where the focus is typically on the four practical PS steps rather than on problem orientation (10). In IPT, PS training aims to help youth understand and resolve relationship problems that may contribute to their depression $(32,33,36,37)$. PS training is also a common component of family therapy (38), cognitive reminiscence therapy (39), and adventure therapy for youth depression (40). The extent to which PS training in these contexts follows the conceptual model by D'Zurilla and colleagues varies. Hereafter, we will distinguish more loosely defined PS training from more formalized PST.

PS skills are highly transferable. In an assessment of fit between evidence-based therapy components and everyday coping skills used by school children, PS skills were the third most frequently endorsed skill set in terms of frequency of habitual use and perceived effectiveness, surpassed only by behavioural activation and increased social support (41). PS training is versatile: It can be brief (i.e., involve fewer than 10 sessions) (22), and has been delivered to youth by trained clinicians (29), lay counsellors (30), and via online platforms (28). It can also be adapted for primary care (24). PS training is a prime candidate for a treatment ingredient that deserves greater scrutiny in the context of youth depression. Yet, no systematic evidence synthesis relating to its efficacy and effectiveness in treating youth depression is currently available.

This study had two sequential parts. First, we conducted a mixed-methods scoping review to map the available evidence relating to PS training as an active ingredient for treating youth depression. Youth were defined as aged 14 to 24 years, broadly aligning with United Nation's definitions (42). The scoping review examined different strands of the published and grey literature to: (a) summarize relevant conceptual discussions (included here as part of the introduction); (b) synthesize existing qualitative research findings about youth's experiences with PS training; (c) provide a narrative review of clinical trials assessing the efficacy of free-standing PST; (d) summarize research examining PS-related 


\section{PROBLEM-SOLVING TRAINING}

concepts as predictors, mediators, or moderators of treatment response; and (d) synthesize recommendations made by clinical practice guidelines (CPGs). Following the mapping and narrative review of the available evidence, we conducted an exploratory meta-analysis to examine the overall efficacy of free-standing PST, based on the clinical trials identified in the scoping review. A panel of youth mental health advisors contributed to the contextualization of review findings.

\section{Methods}

\section{Scoping Review}

As this was the first systematic research synthesis pertaining to PS training for youth depression, we used scoping review methodology to provide an initial overview of the available evidence (43). This scoping review was pre-registered on the Open Science Framework (osf.io/z2g4x) and adhered to the Preferred Reporting Items for Systematic Reviews and Meta-Analysis (PRISMA) extension for Scoping Reviews checklist (see the supplementary materials) (44). The review integrated five types of literature: (a) theoretical studies providing definitions or conceptual discussions (included here as part of the introduction); (b) qualitative studies reporting on youth experiences with PS training; (c) controlled clinical trials testing the efficacy of stand-alone PST; studies examining PS-related concepts as predictors, moderators, or mediators of treatment response; and (e) clinical practice guidelines (CPGs) for youth depression. Approaches to screening, data extraction and synthesis were tailored to each literature type $(45,46)$.

\section{Search Strategy}

We conducted two searches to retrieve theoretical and empirical studies (Search A) and CPGs (Search B) from five bibliographic databases and grey literature sources (see Table 1). We included international literature by considering records published in English, French, German, and Spanish. The strategy for Search A combined topic-specific terms defining the target population and intervention with methodological search filters that combined database-specific subject headings and recommended search terms. The full search strategy is provided in the supplementary materials. Relevant empirical studies were searched for pertinent conceptual discussions; reference lists of key studies were searched manually for additional studies; and records citing key studies were searched using Google Scholar (47). For Search B, we included eligible CPGs from a previous review of English-language CPGs published until July 2015 (Search B.1) $(48,49)$. A research librarian reviewed and expanded this search regarding publication language, bibliographic databases, and publication period to align with the parameters for Search A (see Table 1). The grey literature search used a multipronged approach to retrieve relevant records from common grey literature databases as well as CPG repositories, and by searching websites of relevant associations, charities, and government agencies (see the supplementary materials). 
PROBLEM-SOLVING TRAINING

Table 1. Overview of Systematic Search Parameters

\begin{tabular}{lllll}
\hline & Literature types & Databases & Publication date & Language of publication \\
\hline Search A & $\begin{array}{l}\text { Theoretical \& } \\
\text { empirical research }\end{array}$ & $\begin{array}{l}\text { APA PsycINFO, CINAHL, Embase, } \\
\text { MEDLINE, Web of Science }\end{array}$ & $\begin{array}{l}\text { Database inception-23 } \\
\text { June 2020 }\end{array}$ & $\begin{array}{l}\text { English, French, German, } \\
\text { or Spanish }\end{array}$ \\
$\begin{array}{l}\text { Search B.1 } \\
\text { (original) }(48,49)\end{array}$ & CPGs & $\begin{array}{l}\text { APA PsycINFO, CINAHL, Embase, } \\
\text { MEDLINE }\end{array}$ & $\begin{array}{l}\text { January 2005—July } \\
2015\end{array}$ & English \\
$\begin{array}{l}\text { Search B.2 } \\
\text { (expanded) }\end{array}$ & CPGs & APA PsycINFO, CINAHL, Embase, & January 2005-July & $\begin{array}{l}\text { English, French, German, } \\
\text { or Spanish }\end{array}$ \\
\hline
\end{tabular}

\section{Inclusion and Exclusion Criteria}

Empirical studies were included if the mean participant age fell within the eligible range of 14 to 24 years, and at least $50 \%$ of participants showed above-threshold depressive or emotional symptoms on a validated screening tool. Studies further had to be: (a) a qualitative study examining youth experiences with or perspectives on PS training; (b) a controlled trial examining the effectiveness of PST as a free-standing intervention compared with a control group; or (c) a secondary analysis of data from a controlled trial examining the influence of PS ability or receipt of PS training on treatment response within a broader therapeutic intervention (e.g., CBT). Records were included as CPGs if labelled as practice guidelines, practice parameters, or consensus or expert committee recommendations, or explicitly aimed to develop original clinical guidance $(48,49)$. CPG had to focus on indicated psychosocial treatments for youth depression, rather than screening and assessment, or psychopharmacological treatment. Doctoral dissertations were included. Conference abstracts, non-controlled trials, and studies focusing on prevention rather than indicated treatment were excluded.

\section{Screening}

All records identified were imported into the systematic review software EPPI-Reviewer 4.0 (50). Following the removal of duplicates, records underwent a two-stage screening process to appraise their eligibility for inclusion (Fig 2). Title and abstract screening was conducted by KRK for all studies and duplicated by a second reviewer (DC and BWCC) for $10 \%$ of the identified records, yielding substantial inter-rater agreement ( $k a p p a=.75$ for empirical studies; $k a p p a=$ .86 for CPGs). All studies retained for full text screening were screened by KRK, and $20 \%$ were screened in duplicate by BWCC, yielding substantial agreement ( $k a p p a=.68$ for empirical studies; kappa $=.71$ for CPGs). Disagreements were resolved by discussion.

\section{Data Extraction and Synthesis}

Data extraction involved the use of templates tailored to each literature type (e.g., the Cochrane data collection form for RCTs). Information extracted included: citation details; study type and design; participant sample; approaches to measurement and analysis; relevant qualitative or quantitative data. Additional information extracted from CPGs 


\section{PROBLEM-SOLVING TRAINING}

included the issuing authority, the age range of the target population, the treatment settings to which the guideline applied (e.g., primary care versus specialist services), and any recommendations in relation to PS training. Data from clinical trials and secondary analyses were extracted in duplicate, and any discrepancies were discussed and resolved.

Data synthesis followed a five-step process of data reduction, display, comparison, conclusion drawing, and verification (46). Scoping review findings were summarized in narrative format. In addition, effect sizes reported in PST trials for depression severity were entered into a meta-analysis (see below). To incorporate a variety of perspectives, the wider review team convened for an inference workshop where emerging findings were discussed and interpreted. The multidisciplinary team involved a methodologist; two child and adolescent psychiatrists with expertise in CBT, DBT, and IPT; a psychologist with expertise in parent-adolescent therapy; a research librarian; a family doctor; a biostatistician; a clinical epidemiologist; two youth research partners; and a youth engagement coordinator.

\section{Fig 2. PRISMA Flow Chart of the Study Selection Process}

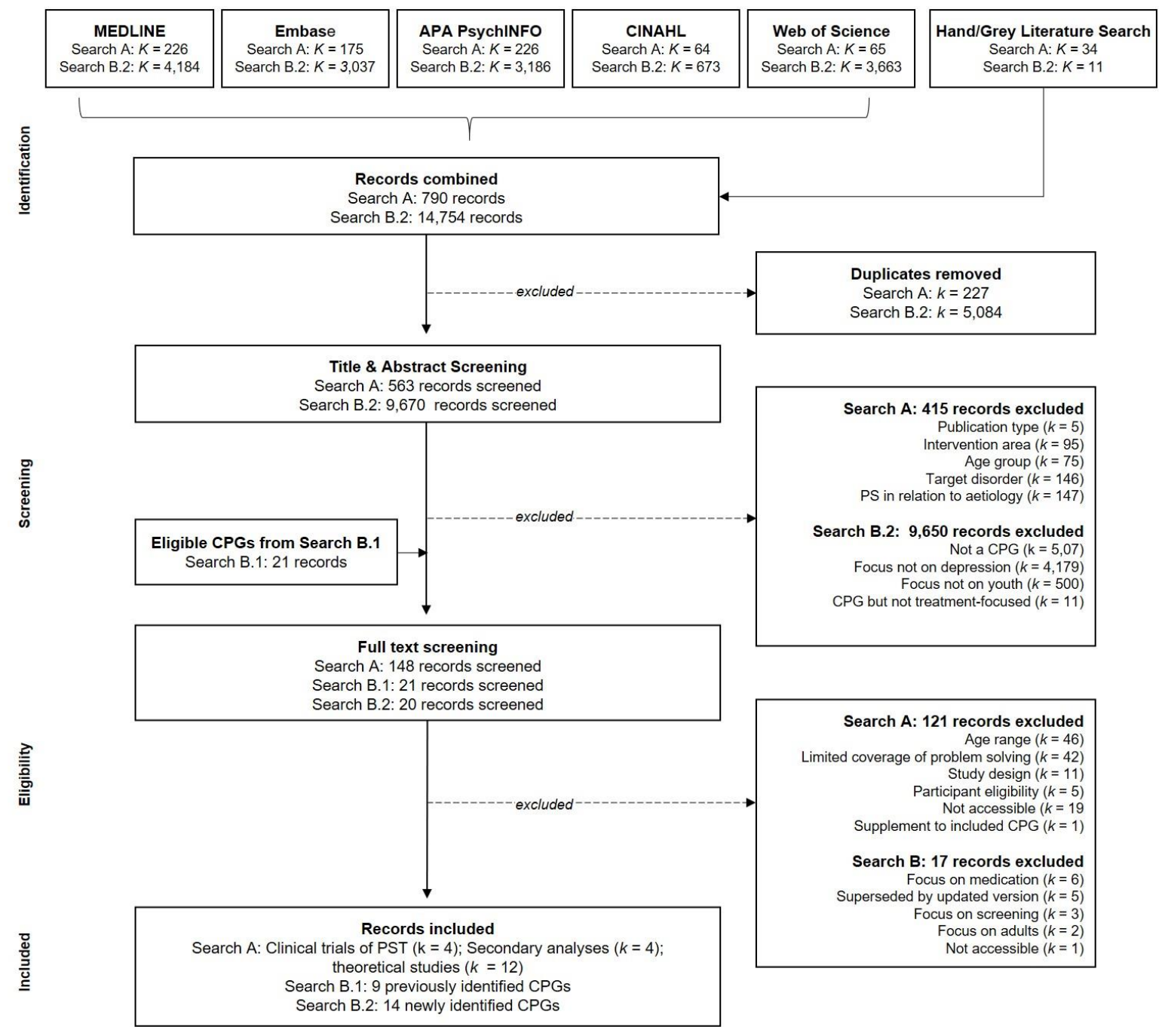




\section{PROBLEM-SOLVING TRAINING}

\section{Meta-Analysis}

Meta-analyses are not typical components of scoping reviews (51). We chose to conduct an exploratory metaanalysis following our scoping review and narrative synthesis of the existing evidence, to obtain an initial indication of the efficacy of stand-alone PST. This meta-analysis was not part of the pre-registered scoping review protocol. For the purpose of the meta-analysis, we assessed the risk of bias for each of the four PST trials using the Cochrane Collaborations ROB 2 tool (see supplementary materials) (52). Ratings were performed independently by two reviewers (KRK and MA), and consensus on the final ratings was formed through discussion. A detailed record of ROB 2 ratings for each study is provided in Tables S3 and S4 in the supplementary materials.

Treatment effect sizes (Hedges' g) and their confidence intervals were calculated based on the mean difference in depression severity scores between the PST and control conditions at the first post-treatment assessment (53). Effect sizes of 0.2 indicate a small effect; effect sizes around 0.5 indicate a moderate effect, and effect sizes of 0.8 are considered large. Hedges' $\mathrm{g}$ is calculated by subtracting the post-treatment mean score of the intervention group from the score of the control group, and by dividing the mean difference by the pooled standard deviation. We adjusted these effect sizes using the Hedges and Olkin small sample correction (53).

Pooled mean effect sizes were computed using the meta suit of commands in Stata 16.1, in a random effects model to account for clinical heterogeneity among the identified studies (e.g., in relation to the intervention setting, mode of delivery, depression severity of the target group, and participant ages). We computed the $I^{2}$ statistic indicating the percentage of variance in the meta-analysis attributable to effect size heterogeneity. While exact interpretation thresholds can be misleading due to the multifactorial nature of heterogeneity, it is suggested that $I^{2}$ below 30\% represents low heterogeneity while $I^{2}$ above $75 \%$ represents substantial heterogeneity (54). Investigations of heterogeneity are unlikely to generate valuable insights if the number of available studies is too small, with at least ten studies recommended for meta-regression (54). We conducted limited exploratory subgroup analysis, by computing a separate effect size after excluding studies with high risk of bias. We inspected the funnel plot and conducted Egger's test to examine the likelihood and extent of publication bias (55).

To characterize the quality of the evidence, we conducted a Grading of Recommendations Assessment, Development, and Evaluation (GRADE) appraisal using the GRADEpro software (56). We graded the evidence for risk of bias, imprecision, indirectness, inconsistency, and publication bias (57). A GRADE of "high quality" indicates a high level of confidence that the true effect lies close to the estimate; "moderate quality" indicates moderate confidence in the estimate (i.e., the true effect is likely close to the estimate although there is a possibility that the true effect is different); "low quality" indicates limited confidence (i.e., the true effect may be substantially different from the estimate); and "very low quality" indicates very little confidence in the estimate (i.e., the true effect is likely to be substantially different). 
PROBLEM-SOLVING TRAINING

\section{Youth Engagement}

The Centre for Addiction and Mental Health (CAMH) implements a Youth Engagement Initiative that brings the voices of youth aged 14 to 29 years with lived experience of mental health difficulties into research and service design (58-60). Two youth partners were co-investigators in this review and led an informal consultation with a panel of twelve CAMH youth advisors to inform the review process and help contextualize results. Formal approval by a Research Ethics Board was not required, as youth were research partners rather than research subjects and provided no individual data.

\section{Results}

\section{Selection and Inclusion of Studies}

Search A for empirical and theoretical studies identified 563 unique records (Fig 2), of which 148 were considered for full text screening. Inclusion criteria were met by twelve theoretical studies (which informed the introduction to this article), four RCTs of PST, and four secondary analyses of clinical trials investigating PS-related concepts as predictors, mediators, or moderators of treatment response. We did not identify any qualitative studies that explicitly examined youth experiences of PS training. The updated Search B.2 for CPGs identified 9,672 unique records, and 21 CPGs were added from a previous systematic search (Search B.1) (49). Overall, 40 potential CPGs were subject to full text screening, of which 23 were included in the review. Below we present scoping review findings for all evidence types, followed by the results from our exploratory meta-analysis of stand-alone PST trials.

\section{Clinical Trials of PST}

Characteristics of the included PST trials are summarized in Table 2. All four RCTs were published between 2008 and 2020. They included 524 participants, with sample sizes ranging from 45 to 250 per study. The participant mean age was 16.7 years (range: 12-25 years). Around half of participants were female. One study was targeted at youth with a diagnosis of MDD, one at youth with elevated anxiety or depressive symptoms, and two at youth with various mild presenting problems including depression. In all four studies, treatment covered the four PS skills, but not problem orientation (i.e., youth's problem appraisals). In three studies, treatment was delivered face-to-face, while one employed self-directed PST online. The mean number of planned sessions was 5.5 (range: 5-6 sessions). Two studies compared PST with a wait-list control, one compared PST with booklets to the use of booklets alone, and one compared PST with supportive counselling. Risk of bias was rated as low for one (30), medium for two $(28,29)$, and high for one study (27), with concerns relating to missing outcome data at the post-treatment assessment, and to the absence of a study protocol or analysis plan in one study (30) (see supplementary materials). 
PROBLEM-SOLVING TRAINING

Table 2. Study Characteristics - Clinical Trials of Stand-Alone PST

\begin{tabular}{|c|c|c|c|c|c|c|c|c|c|c|}
\hline Study & Country & $\begin{array}{l}\text { Mean } \\
\text { Age in } \\
\text { Years }\end{array}$ & $\begin{array}{l}\text { Diagnostic } \\
\text { Status }\end{array}$ & $\begin{array}{l}\% \text { with } \\
\text { elevated } \\
\text { depressive } \\
\text { symptoms }\end{array}$ & Recruitment & $N^{\mathrm{a}}$ & Conditions & $\begin{array}{l}N \\
\text { sessions }\end{array}$ & $\begin{array}{l}\text { Outcome } \\
\text { considered for } \\
\text { meta-analysis }\end{array}$ & $\begin{array}{l}\text { Risk of } \\
\text { Bias }\end{array}$ \\
\hline Eskin (27) & Turkey & $\begin{array}{l}\text { N/A } \\
(19.1)\end{array}$ & Diag. (Dep) & 100.0 & Community & 53 & $\begin{array}{l}\text { 1. PST } \\
\text { 2. Waitlist }\end{array}$ & 6 & $\mathrm{BDI}(\mathrm{P})$ & High \\
\hline Hoek (28) & Netherlands & $\begin{array}{l}12-21 \\
(16.1)\end{array}$ & $\begin{array}{l}\text { Elev. } \\
\text { (Anx or Dep) }\end{array}$ & 80.0 & Community & 45 & $\begin{array}{l}\text { 1. PST } \\
\text { 2. Waitlist }\end{array}$ & 5 & CES-D (P) & $\begin{array}{l}\text { Some } \\
\text { concerns }\end{array}$ \\
\hline Parker (29) & Australia & $\begin{array}{l}15-25 \\
(17.6)\end{array}$ & Elev. (various) & 54.1 & Clinical & 176 & $\begin{array}{l}\text { 1. } \mathrm{PST}^{\mathrm{c}} \\
\text { 2. } \mathrm{SUP}^{\mathrm{c}}\end{array}$ & 6 & BDI-II (P) & $\begin{array}{l}\text { Some } \\
\text { concerns }\end{array}$ \\
\hline $\begin{array}{l}\text { Michelson } \\
\text { (30) }\end{array}$ & India & $\begin{array}{l}12-20 \\
(15.6)\end{array}$ & Elev. (various) & $53.0^{b}$ & Community & 251 & $\begin{array}{l}\text { 1. PST } \\
\text { 2. PST }\end{array}$ & 5 & $\begin{array}{l}\text { SDQ } \\
\text { emotional } \\
\text { symptoms (S) }\end{array}$ & Low \\
\hline
\end{tabular}

Note. BDI: Beck Depression Inventory (85); CES-D: Center for Epidemiologic Studies Depression Scale (86); Diag.: Diagnosis; Elev.: elevated symptoms; P: defined as a primary outcome in the primary study; SDQ = Strengths and Difficulties Questionnaire (87); S: defined as a secondary outcome in the primary study; SUP: supportive counselling.

${ }^{\text {a }}$ The total sample size reported is the number of participants randomized to intervention and control conditions.

${ }^{\mathrm{b}}$ Refers to emotional symptoms, rather than depressive symptoms, specifically.

${ }^{\mathrm{c}}$ Delivered with adjunctive behavioural activation or psychoeducation in a factorial $2 \times 2$ design.

Eskin and colleagues [28] randomized 53 Turkish high school and university students with MDD to six sessions of PST or a waitlist. PST had a significant treatment effect on self-reported depressive symptoms $(\mathrm{d}=-1.20 ; \mathrm{F}[1,42]=$ $10.3, p<.01$. $)$ and clinician-reported depressive symptoms $(\mathrm{d}=-2.12 ; \mathrm{F}[1,42]=37.7, p<.001)$, but not on self-reported PS ability $(\mathrm{d}=-0.46 ; \mathrm{F}[1,42]=2.2, p>.05)$. Participants in the PST condition had significantly higher recovery rates in self-reported $(\mathrm{z}=3.76, p<.001)$ and clinician-reported depressive symptoms $(\mathrm{z}=5.09, p<.001)$. Risk of bias was rated as high due to $37 \%$ of missing outcome data in the control group and the absence of a published trial protocol.

Michelson and colleagues (30) compared PST delivered by lay counsellors in combination with printed booklets, to printed booklets alone. Participants were 251 high-school students from low-income communities in New Delhi, India, with mild mental health symptoms, including emotional problems (53\%). PST with booklets was significantly more effective at reducing self-reported priority problems at six weeks $(\mathrm{d}=0.36, p=.0015)$ and twelve weeks $(\mathrm{d}=0.35, p$ $=.0004)$ post intake than the control. However, PST was not significantly more effective at reducing self-reported mental health problems at six $(d=0.16, p=.18)$ or twelve weeks $(d=0.20, p=.07)$, or self-reported emotional symptoms at 12 weeks $(\mathrm{d}=0.18, p=.089)$. As there was no long-term follow-up, it is unknown whether reduced personal problems translated into reduced emotional symptoms in the longer term. Baseline symptom chronicity, severity, and youth top problem type were not significant treatment moderators. Perceived stress at six weeks was found to mediate treatment effect on idiographic problems, accounting for $15 \%$ of the overall effect at 12 weeks. No adverse effects were reported. 


\section{PROBLEM-SOLVING TRAINING}

Two trials found no significant effect of PST on primary or secondary outcomes: Hoek and colleagues (28) randomized 45 youth with elevated depression or anxiety symptoms to five sessions of online PST or a waitlist control; Parker and colleagues (29) randomized 176 youth with mixed presenting problems including depression (54\%) to either PST with physical activity or PST with psychoeducation, compared with supportive counselling with physical activity or psychoeducation (29). Drop-out from PST was high in both studies, ranging from $41.4 \%$ (29) to $72.7 \%$ (28).

\section{PS-Related Concepts as Predictors, Moderators, or Mediators of Treatment Response}

We identified four secondary analyses of RCT data that examined PS-related concepts as predictors, moderators, or mediators of treatment response (see Table 3). The studies were published between 2005 and 2014 and included 929 participants with a mean age of 15.2 years (range: $12-18$ years), of which $61.2 \%$ were female. All four trials required a diagnosis of major depressive disorder (MDD).

Becker-Weidman and colleagues (61) examined data from the 439 participants of the Treatment for Adolescents With Depression Study (TADS) (62). Here, they explored whether baseline problem orientation and PS styles, as measured by the Social Problem-Solving Inventory-Revised (SPSI-R) (63), were significant predictors or moderators of treatment response (61). Negative problem orientation and avoidant PS style at baseline each predicted less improvement in depression symptom severity by the 12-week endpoint ( $p=.001$ and $p=0.003$, respectively); positive problem orientation at baseline predicted greater improvement $(p=.002)$. These variables did not moderate the relationship between treatment condition and change in depression symptom severity. Neither rational PS nor impulsivecareless PS styles at baseline predicted or moderated change in depressive symptoms over time.

Kennard and colleagues (64) examined data from 334 youth with treatment-resistant depression who participated in the Treatment of Resistant Depression in Adolescents (TORDIA) trial (65). CBT participants who received PS training were 2.3 times $(p=.03)$ more likely to have a positive treatment response than those not receiving this component as part of CBT. A similar effect was observed for social skills training ( $\mathrm{OR}=2.6, p=.04)$, but not for seven other CBT components. However, PS and social skills training had the most equal allocation ratios between youth who did and did not receive them out of all CBT components (51.8\% of youth received PS training; $54.2 \%$ received social skills training). Balanced allocation ratios provide maximum power for a given sample size, while power decreases with growing imbalance (66). Analysis of the remaining seven components may have been underpowered, as allocation ratios ranged between 1:3 and 1:5. It must also be noted that treatment modules were not randomly assigned but selected based on the clinical needs of individual cases. The authors did not correct for multiple comparisons as part of this exploratory analysis. 
PROBLEM-SOLVING TRAINING

Table 3. Study Characteristics and Findings—-Secondary Analyses Testing PS as a Predictor, Moderator, or Mediator of Treatment Response

\begin{tabular}{|c|c|c|c|c|c|c|c|c|c|c|c|}
\hline Study & $\begin{array}{l}\text { Countr } \\
\mathrm{y}\end{array}$ & $\begin{array}{l}\text { Mean } \\
\text { Age in } \\
\text { Years }\end{array}$ & $\mathrm{N}^{\mathrm{a}}$ & $\begin{array}{l}\text { Diagnostic } \\
\text { Status }\end{array}$ & $\begin{array}{l}\text { Recruitment } \\
\text { Setting }\end{array}$ & $\begin{array}{l}\text { Overarching } \\
\text { intervention(s) }\end{array}$ & $\begin{array}{l}\text { Effect } \\
\text { type }\end{array}$ & $\begin{array}{l}\text { Operationalization of } \\
\text { problem solving }\end{array}$ & Outcome & PS measure & Effect \\
\hline $\begin{array}{l}\text { Becker- } \\
\text { Weidman } \\
\text { (61) }\end{array}$ & USA & $\begin{array}{l}12-17 \\
(14.6)\end{array}$ & 439 & MDD & Clinical & $\begin{array}{l}\text { 1. Fluox } \\
\text { 2. CBT } \\
\text { 3. Fluox }+ \text { CBT } \\
\text { 4. Placebo } \\
\text { (TADS) }\end{array}$ & $\begin{array}{l}\text { Pred / } \\
\text { Mod }\end{array}$ & $\begin{array}{l}\text { Baseline PPO, NPO, AS, } \\
\text { ICS, RPS, }\end{array}$ & $\begin{array}{l}\text { Depression } \\
\text { severity } \\
\text { (CDRS-R) }\end{array}$ & SPSI-R (63) & $\begin{array}{l}\text { NPO, PPO, AS = } \\
\text { Pred** }^{* *}\end{array}$ \\
\hline Kennard (64) & USA & $\begin{array}{l}12-18 \\
(16.0)\end{array}$ & 334 & MDD (TR) & Clinical & $\begin{array}{l}\text { Switch to: } \\
\text { 1. Diff SSRI } \\
\text { 2. Diff. SSRI + CBT } \\
\text { 3. Ven } \\
\text { 4. Ven + CBT } \\
\text { (TORDIA) }\end{array}$ & Pred & $\begin{array}{l}\text { Receipt of PS training as } \\
\text { part of CBT vs. no receipt }\end{array}$ & $\begin{array}{l}\text { Treatment } \\
\text { response } \\
\text { (CDRS-R \& } \\
\text { CGI-I) }\end{array}$ & N/A & $\begin{array}{l}\text { PS training } \\
\text { receipt }=\text { Pred }^{*}\end{array}$ \\
\hline Kaufman (68) & USA & $\begin{array}{l}13-17 \\
(15.1)\end{array}$ & 93 & MDD \& CD & Community & $\begin{array}{l}\text { 1. CWD-A } \\
\text { 2. LST }\end{array}$ & Med & $\begin{array}{l}\Delta \text { self-reported PS in } \\
\text { specific situations }\end{array}$ & $\begin{array}{l}\text { Depression } \\
\text { severity } \\
\text { (BDI-II \& } \\
\text { HDRS) }\end{array}$ & $\begin{array}{l}\text { Issues } \\
\text { Checklist (69) }\end{array}$ & No effect \\
\hline Dietz (67) & USA & $\begin{array}{l}13-18 \\
(15.6)\end{array}$ & 63 & MDD & Clinical & $\begin{array}{l}\text { 1. CBT } \\
\text { 2. SBFT } \\
\text { 3. NST }\end{array}$ & Med & $\begin{array}{l}\Delta \text { observed interpersonal } \\
\text { PS interactions }\end{array}$ & $\begin{array}{l}\text { MDD } \\
\text { remission } \\
\text { (K-SADS \& } \\
\text { BDI). }\end{array}$ & $\begin{array}{l}\text { Coding of } \\
\text { videotaped } \\
\text { youth-mother } \\
\text { interactions }\end{array}$ & No effect \\
\hline
\end{tabular}

Note. $\Delta$ : Change in; AS: Avoidance Style; BDI: Beck Depression Inventory (88); CDRS-R: Children's Depression Rating Scale—Revised (89); CGI-I: Clinical Global Impression Scale—-Improvement (90); CWD-A: Adolescent Coping with Depression Course; Diff: different; Fluox: Fluoxetine; ICS: Impulsivity/Carelessness Style; K-SADS: The Kiddie Schedule for Affective Disorders and

Schizophrenia (91); LST: Lifeskills training; MDD: Major Depressive Disorder; Med: Mediator; Mod: Moderator; NPO: Negative Problem Orientation; NST: Nondirective supportive therapy; PPO:

Positive Problem Orientation; Pred: Predictor; RPS: Rational Problem Solving; SBFT: Systemic Behaviour Family Therapy; SPSI-R: Social Problem-Solving Inventory Revised (63); Ven: Venlafaxine.

$* p<.05 ; * * p<.01 ; * * * p<.001$

${ }^{a}$ The total sample size reported is the number of participants randomized to intervention and control conditions. 


\section{PROBLEM-SOLVING TRAINING}

Dietz and colleagues (67) examined data from an RCT comparing CBT, Systemic Behaviour Family Therapy (SBFT), and Non-Directive Supportive Therapy (NST) in a sample of 63 depressed youth. CBT focused on cognitive restructuring, while also providing PS training and behavioural activation on a case-by-case basis. SBFT provided PS training to reduce family conflict, alongside functional family therapy. Both interventions were significantly associated with improvements in adolescents' interpersonal PS (as measured by coding videotaped interactions between adolescents and their mothers) over the course of treatment (CBT: $\mathrm{b}^{*}=0.41, p=.006$; SBFT: $\left.\mathrm{b}^{*}=0.30, p=.04\right)$. Improvements in adolescent PS interactions were significantly associated with higher rates of remission post treatment (Wald $\mathrm{z}=6.11, p$ $=.01)$. There was no significant indirect effect of change in youth PS interactions on remission status, and hence, no definitive evidence that PS significantly mediated treatment effectiveness.

Kaufman and colleagues (68) examined data from a trial comparing an Adolescent Coping with Depression (CWD-A) group-based intervention with a life-skills control condition in 93 youth with comorbid depression and conduct disorder. The study examined whether six CBT-specific factors (i.e., use of social skills, relaxation skills, and PS and conflict resolution skills; frequency of engagement in pleasant activities; frequency and magnitude of negative thoughts and dysfunctional attitudes) mediated the effectiveness of CWD-A. There was no significant improvement in PS ability in CWD-A, compared with the control condition, as measured via selected items from the Issues Checklist (69), and hence no further analysis was conducted to examine its role as a mediator.

\section{PS Training in Clinical Practice Guidelines}

We identified 23 CPGs from twelve countries relevant to youth depression (see supplementary materials). Issuing bodies were governments $(k=6)$, specialty societies $(k=3)$, health care providers $(k=4)$, independent expert groups $(k$ $=2$ ), and others $(k=4)$, or a combination of these. Of these 23 CPGs, 15 mentioned PS training in relation to depression treatment for youth. PS training was described as a component of CBT $(k=7)$, IPT $(k=4)$, supportive therapy $(k=3)$, family therapy $(k=1)$, DBT $(k=1)$, and psychoeducation $(k=1)$.

No CPG recommended free-standing PST as a first-line treatment for youth depression. However, five CPGs mentioned PS training in the context of recommending broader therapeutic approaches. The World Health Organization's updated Mental Health Gap Action Programme (mhGAP) guidelines recommended PS training as an adjunct treatment (e.g., in combination with antidepressant medication) for adults and older adolescents (70). A guideline issued by Orygen (Australia) based on recommendations by the National Institute for Health and Care Excellence (NICE) recommended that for "persistent sub-threshold depressive symptoms (including dysthymia) or mild to moderate depression", options should include "6-8 sessions of individual guided self-help based on the principles of CBT, including behavioural activation and problem-solving techniques" (71). A guideline issued by the Chilean Ministry of Health for individuals aged 15 and older suggested that for mild depression, the most effective treatment would be supportive clinical care with 


\section{PROBLEM-SOLVING TRAINING}

adjunctive psychoeducation and PS tools, or supportive counselling (p. 52) (72). A fourth guideline issued by the Cincinnati Children's Hospital Medical Centre recommended four to eight sessions of supportive therapy for mild or uncomplicated depression, adding that "problem solving coping skills" were one element of supportive therapy (p. 1) (73). They referred to an earlier practice parameter by Birmaher and Brent (74), which suggested that each phase of treatment for child and youth depression should include supportive management (alongside psychoeducation), and that this might include PS training (p. 1510). None of these CPGs specified whether PS training should incorporate specific modules, or whether the term was used loosely to describe unstructured PS support.

\section{Meta-Analysis}

Each of the four RCTs of free-standing PST identified by the scoping review contributed one comparison to the exploratory meta-analysis of overall PST efficacy (see Fig 3). Self-rated depression severity scores were reported by all four studies and constituted the primary outcome in this meta-analysis. We conducted additional exploratory analysis for clinician-rated depression severity as reported in two studies $(27,29)$. The pooled effect size for self-reported depression severity was $\mathrm{g}=-0.34$ (95\% CI: -0.92 to 0.23$)$. Heterogeneity was high $\left(I^{2}=88.37 \% ; p<.001\right)$. Examination of the funnel plot (see Fig S1 in the supplementary materials) and Egger's test $(\beta=-3.93 ; p=.19)$ suggested no publication bias. In order to achieve the best possible estimate of the true effect size we computed a second model including only studies with low to medium risk of bias (i.e., three studies), thus excluding the only study showing a large and significant effect (i.e., 27). The resulting effect size was $\mathrm{g}=-0.08$ (95\% CI: -0.26 to 0.10$)$, with no significant heterogeneity $\left(I^{2}=0.00 \% ; p=\right.$ 0.72; see Fig S3 in the supplementary materials). The pooled effect size for clinician-rated depression severity was $\mathrm{g}=$ 1.39 with a very wide confidence interval (95CI: -4.03 to 1.42$)$ and very high heterogeneity $\left(I^{2}=97.41 \%, p<0.001\right.$; see Fig S2 in the supplementary materials).

Fig 3. Forest Plot: Random Effects Model with Self-Reported Depression or Emotional Symptoms (Continuous) as Primary Outcome

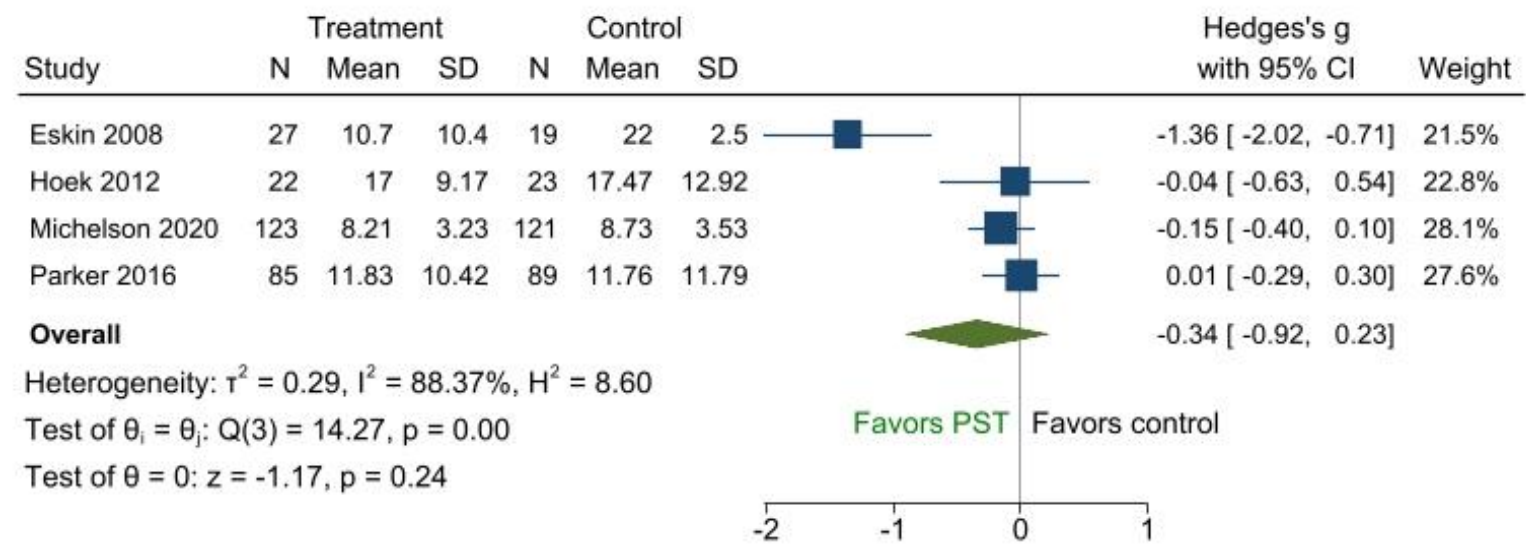

Random-effects REML model 


\section{PROBLEM-SOLVING TRAINING}

The results of the GRADE assessment for the primary outcome of self-reported depression severity across all four studies, as well as secondary outcomes are shown in Table S5 in the supplementary material. The quality of the evidence supporting the primary outcome was very low, with concerns relating to risk of bias, inconsistency, indirectness, and imprecision. Quality ranged from low to very low for secondary outcomes.

\section{Youth Insights}

Twelve CAMH youth advisors with lived experience helped contextualize the review findings by providing their insights into PS. Emerging review findings were not shared with the panel prior to the consultation, so as not to steer their inputs. Most youth advisors had participated in PS training as part of other therapies, but none had received formal PST. A key challenge identified was how to provide PS training that is universally applicable and relevant to different youth without being too generic or rigid, and that could accommodate youth perspectives, complex problems, and individual situations and dispositions. Youth advisors suggested PS training should be (a) youth-driven, (b) strengths-based, (c) comprehensive, and (d) personalized—as summarized in Fig 4.

Fig 4. Principles for PS Training Suggested by Youth Advisors

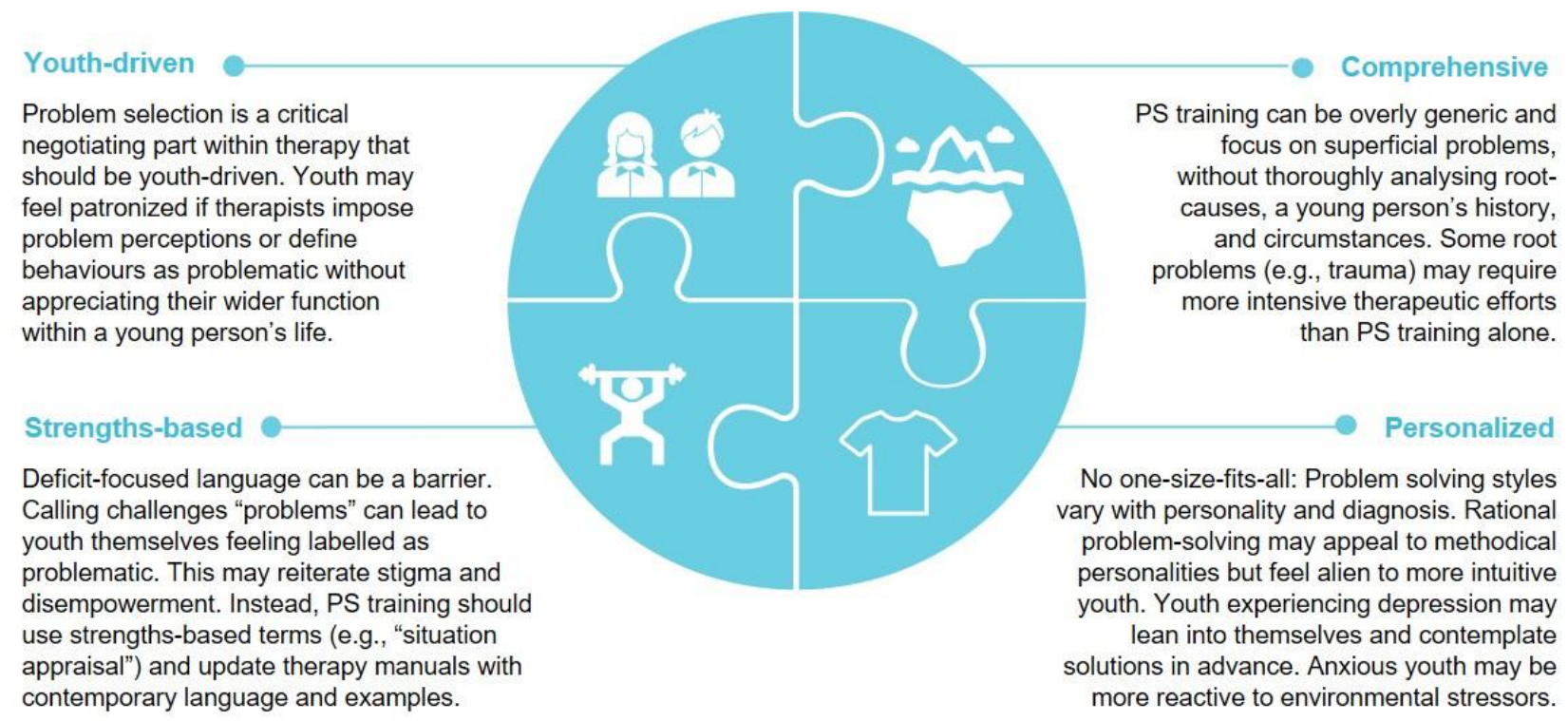

\section{Discussion}

This scoping review aimed to provide an overview of the evidence relating to PS training as an active ingredient for treating youth depression. The scoping review and exploratory meta-analysis of four RCTs found no strong evidence supporting the use of stand-alone PST for treating youth depression. The scoping review identified some evidence that PS training may enhance treatment response in CBT, but this conclusion was drawn from secondary analyses, where youth were not randomized to treatment with and without PS training, and where primary studies were not powered to 


\section{PROBLEM-SOLVING TRAINING}

test these differences. Disproportionate exposure to comparator CBT components also limits these findings. PST was not recommended as a stand-alone treatment for youth depression in 24 CPGs; however, one guideline suggested it could be provided alongside other treatments (e.g., antidepressants) for older adolescents, and four suggested PS training as a component of low-intensity psychosocial interventions for youth with mild to moderate depression. Input from youth advisors suggested that PS training can be helpful if it is youth-driven, comprehensive, personalized, and strengths-based. Youth advisors emphasized that PS training should identify the root causes underpinning superficial problems and address these through suitable complementary approaches.

Given the limited evidence base, only tentative suggestions can be made as when and for whom PS training is effective. The one PST trial with a low risk of bias enrolled high-school students from low-income communities in New Delhi, and found that PST delivered by lay counselors in combination with printed booklets was more effective at reducing youth's personal priority problems than printed booklets alone, without effectively reducing mental health symptoms (30). Within a needs-based framework of service delivery (e.g., 75), PST may be offered as a low-intensity intervention to youth who experience challenges and struggle with PS-including in low-resource contexts. Future research could explore whether PS training might be particularly helpful for youth facing socioeconomic hardship and related chronic stressors by protecting them from the potentially harmful effects of these stressors on well-being (76). If findings are promising, PS training may be considered for targeted prevention (e.g., 26). However, at this time there is insufficient evidence to support PS training on its own as an intervention aimed at providing symptom relief for youth experiencing depression.

The PST manual suggests cognitive overload, emotional dysregulation, negative thinking and hopelessness interfere with PS (9). Youth whose depression hinders their ability to engage in PST may require additional support through more comprehensive therapy packages such as CBT or IPT with PS training. In the TORDIA trial (65), where PS training was found to be one of the most effective components, it was generally taught alongside cognitive restructuring, behavioural activation, and emotion regulation, which may have facilitated youths' ability to absorb PS training (64). The focus of these other CBT components on changing negative cognitions and attributions may fulfil a similar function as problem orientation modules in stand-alone PST. Research that is powered to explore such mechanisms is needed. In addition, future research should apply methodologies designed to identify the most critical elements in a larger treatment package (e.g., dismantling studies; or sequential, multiple assignment, randomized trials) to examine the role of PS training when delivered alongside other components. While one trial focusing on CBT components is currently underway (77), similar research is needed for other therapies (e.g., IPT, DBT, family therapy).

The included PST trials provided between five and six sessions and covered PS skills but not problem orientation. Meta-analyses of PST for adult depression suggest treatment effectiveness may be enhanced by longer treatment duration 


\section{PROBLEM-SOLVING TRAINING}

( $\geq 10$ sessions) (22), and coverage of problem orientation alongside PS skills (23). As per the PST treatment manual, strengthening problem orientation fosters motivation and self-efficacy and is an important precondition for enhancing skills (78,79). In addition, only one youth PST trial assessed PS ability at baseline (27). A meta-analysis of PST for adult depression (23) suggests that studies including such assessments show larger effect sizes, with therapists better able to tailor PST to individual needs. Future research should seek to replicate these findings specifically for youth depression.

Youth stakeholders suggested reviewing and reworking PS training with youth in mind. Since its development in the 1970s, PST has undergone several revisions $(9,78,80-82)$ but tailoring to youth has been limited. Designing and updating therapy manuals in consultation with youth, and promoting strengths-based language is imperative (58). Solution-focused brief therapy (SFBT) has emerged as an antithesis to PST where the focus is on constructing solutions rather than solving problems, and service users are encouraged to envisage how they would like their life to be, with little time spent on analysing problems (31). SFBT may be more consistent with youth preferences for strengths-based approaches but may provide insufficiently comprehensive problem appraisals. Future research should compare the effectiveness and acceptability of PST and SFBT, and consider possible benefits of combining the strengths of both approaches, to provide support that is strengths-based and targets root problems.

\section{Strengths and Limitations}

This scoping review applied a broad and systematic approach to study identification and selection. We searched five bibliographic databases, and conducted an extensive grey literature search, considering records published in four languages. Nevertheless, our search may have missed relevant studies conducted in other languages. We found only a small number of eligible empirical studies, several of which were likely underpowered. As stated above, studies analysing PS-related concepts as predictors, moderators, or mediators of treatment response within broader therapies were heterogenous and limited by design and sample size constraints.

Similarly, there was heterogeneity in recruitment and intervention settings, age groups, and delivery formats across the four RCTs of stand-alone PST, and the overall quality of the evidence was very low. As reflected in our GRADE appraisal, one important limitation was the indirectness of the available evidence: Only one PST trial focused specifically on youth with an MDD diagnosis, while the remaining three included youth with a mix of mental health problems. Although outcomes were reported in terms of depression or emotional symptom severity, this was not based on a subgroup analysis focused specifically on youth with depression. Impact on this group may therefore have been underestimated. In addition, the only PST trial with a low risk of bias did not administer a dedicated depression symptom scale. Instead, our exploratory meta-analysis included scores from the 5-item SDQ emotional problems subscale, which assesses unhappiness, worries, clinginess, fears, and somatic symptoms - and may not have captured nuanced change in depression severity $(83,84)$. Other concerns that led us to downgrade the quality of the evidence related to considerable 


\section{PROBLEM-SOLVING TRAINING}

risk of bias, with only one out of four studies rated as having a low risk; and imprecision with several studies involving very small samples. Due to the small number of eligible studies, it was not possible to identify the factors driving treatment efficacy via meta-regression. The long-term effectiveness of PS training, or the conditions under which long-term benefits are likely to be realized also could not be examined (22).

\section{Conclusions}

PS training is a core component of evidence-based therapies for youth depression and described as such by several CPGs. Yet, the evidence base supporting its efficacy as a stand-alone treatment is limited and of low quality. Similarly, there is only tentative evidence suggesting PS-training may drive positive outcomes when provided alongside other components. On its own, PS training may be beneficial for youth with PS deficits who are not acutely distressed or impaired but require support with tackling personal problems. Youth experiencing elevated depressive symptoms may require more comprehensive psychotherapeutic support, as there is currently no robust evidence for the ability of freestanding PST to effectively reduce depressive symptomatology. High-quality trials are needed that assess PST efficacy in youth with mild, moderate, and severe depression, in relation to both symptom severity and idiographic treatment goals or priority problems. These studies should examine the influence of treatment length and module content on treatment impact. Dedicated studies are needed to shed light on the role of PS training as an active ingredient of more comprehensive therapies such as CBT, DBT, IPT, and family therapy. This should include assessment of adverse events and of cost effectiveness. Given high drop-out rates in several youth PST trials, it is important to adapt PS training approaches as needed, following a youth-engaged research and service development approach, to ensure their relevance and acceptability to this age group. 


\section{References}

1. World Health Organization. Adolescent mental health [Internet]. 2019 [cited 2019 Dec 3]. Available from: https://www.who.int/news-room/fact-sheets/detail/adolescent-mental-health

2. Zhou X, Hetrick SE, Cuijpers P, Qin B, Barth J, Whittington CJ, et al. Comparative efficacy and acceptability of psychotherapies for depression in children and adolescents: A systematic review and network meta-analysis. World Psychiatry. 2015;14(2):207-22.

3. Zhou X, Teng T, Zhang Y, Del Giovane C, Furukawa TA, Weisz JR, et al. Comparative efficacy and acceptability of antidepressants, psychotherapies, and their combination for acute treatment of children and adolescents with depressive disorder: a systematic review and network meta-analysis. The Lancet Psychiatry [Internet]. 2020;7(7):581-601. Available from: http://dx.doi.org/10.1016/S2215-0366(20)30137-1

4. Bear HA, Edbrooke-Childs J, Norton S, Krause KR, Wolpert M. Systematic Review and Meta-analysis: Outcomes of Routine Specialist Mental Health Care for Young People With Depression and/or Anxiety. J Am Acad Child Adolesc Psychiatry [Internet]. 2020 Jul;59(7):810-41. Available from: https://linkinghub.elsevier.com/retrieve/pii/S0890856719322348

5. Cuijpers P, Stringaris A, Wolpert M. Treatment outcomes for depression: challenges and opportunities. The Lancet Psychiatry [Internet]. 2020 Feb;0366(2019):2019-20. Available from: http://dx.doi.org/10.1016/S22150366(20)30036-5

6. Kazdin AE. Understanding how and why psychotherapy leads to change. Psychother Res. 2009;19(4-5):418-28.

7. de Haan AM, Boon AE, de Jong JTVM, Hoeve M, Vermeiren RRJM. A meta-analytic review on treatment dropout in child and adolescent outpatient mental health care. Clin Psychol Rev. 2013;33(5):698-711.

8. Chorpita BF, Daleiden EL. Mapping Evidence-Based Treatments for Children and Adolescents: Application of the Distillation and Matching Model to 615 Treatments From 322 Randomized Trials. J Consult Clin Psychol. 2009;77(3):566-79.

9. Nezu AM, Nezu CM, D’Zurilla TJ. Problem-solving therapy: A treatment manual. Problem-solving therapy: A treatment manual. Springer Publishing Company; 2013. x, 323-x, 323.

10. Ugueto AM, Santucci LC, Krumholz LS, Weisz JR. Problem-Solving Skills Training. In: Sburlati ES, Lyneham HJ, Schniering CA, Rapee RM, editors. Evidence-Based CBT for Anxiety and Depression in Children and Adolescents: A Competencies-Based Approach. Chichester, UK: John Wiley \& Sons; 2014. p. 247-59.

11. D'Zurilla TJ, Nezu AM. Problem-solving therapy. In: Handbook of Cognitive-Behavioral Therapies. Third. New York, NY: Guilford Press; 2010. p. 197-225.

12. D'Zurilla TJ, Chang EC, Nottingham IV EJ, Faccini L. Social problem-solving deficits and hopelessness, depression, and suicidal risk in college students and psychiatric inpatients. J Clin Psychol. 1998;54(8):1091-107.

13. Haugh JA. Specificity and social problem-solving: Relation to depressive and anxious symptomology. J Soc Clin Psychol. 2006;25(4):392-403.

14. Nezu AM. Cognitive appraisal of problem solving effectiveness: Relation to depression and depressive symptoms. J Clin Psychol. 1986;42(1):42-8.

15. Reinecke MA, Dubois DL, Schultz TM. Social problem solving, mood, and suicidality among inpatient adolescents. Cognit Ther Res. 2001;25(6):743-56.

16. Siu AMH, Shek DTL. Social problem solving as a predictor of well-being in adolescents and young adults. Soc Indic Res. 2010;95(3):393-406.

17. Ng CSM, Hurry J. Depression Amongst Chinese Adolescents in Hong Kong: An Evaluation of a Stress Moderation Model. Soc Indic Res. 2011;100(3):499-516.

18. D'Zurilla TJ, Nezu AM, Maydeu-Olivares A. Social Problem Solving: Theory and Assessment. In: Chang EC, D'Zurilla TJ, Sanna LJ, editors. Social problem solving: Theory, research, and training. American Psychological 


\section{PROBLEM-SOLVING TRAINING}

Association; 2004. p. 11-27.

19. Nezu AM. Problem solving and behavior therapy revisited. Behav Ther. 2004;35(1):1-33.

20. D'Zurilla TJ, Goldfried MR. Problem solving and behavior modification. J Abnorm Psychol. 1971;78(1):10726.

21. Bandura A, Walters RH. Social learning theory. Vol. 1. Prentice-hall Englewood Cliffs, NJ; 1977.

22. Cuijpers P, de Wit L, Kleiboer A, Karyotaki E, Ebert DD. Problem-solving therapy for adult depression: An updated meta-analysis. Eur Psychiatry [Internet]. 2018;48:27-37. Available from: http://dx.doi.org/10.1016/j.eurpsy.2017.11.006

23. Bell AC, D'Zurilla TJ. Problem-solving therapy for depression: A meta-analysis. Clin Psychol Rev [Internet]. 2009;29(4):348-53. Available from: http://dx.doi.org/10.1016/j.cpr.2009.02.003

24. Zhang A, Park S, Sullivan JE, Jing S. The effectiveness of problem-solving therapy for primary care patients' depressive and/or anxiety disorders: A systematic review and meta-Analysis. J Am Board Fam Med. 2018;31(1):139-50.

25. Stark KD, Reynolds WM, Kaslow NJ. A comparison of the relative efficacy of self-control therapy and a behavioral problem-solving therapy for depression in children. J Abnorm Child Psychol. 1987;15(1):91-113.

26. Spence SH, Sheffield JK, Donovan CL. Preventing adolescent depression: An evaluation of the problem solving for life program. J Consult Clin Psychol. 2003;71(1):3-13.

27. Eskin M, Ertekin K, Demir H. Efficacy of a problem-solving therapy for depression and suicide potential in adolescents and young adults. Cognit Ther Res [Internet]. 2008 Apr 23;32(2):227-45. Available from: http://link.springer.com/10.1007/s10608-007-9172-8

28. Hoek W, Schuurmans J, Koot HM, Cuijpers P. Effects of Internet-Based Guided Self-Help Problem-Solving Therapy for Adolescents with Depression and Anxiety: A Randomized Controlled Trial. PLoS One. 2012;7(8):17.

29. Parker AG, Hetrick SE, Jorm AF, Mackinnon AJ, McGorry PD, Yung AR, et al. The effectiveness of simple psychological and physical activity interventions for high prevalence mental health problems in young people: A factorial randomised controlled trial. J Affect Disord [Internet]. 2016;196:200-9. Available from: http://dx.doi.org/10.1016/j.jad.2016.02.043

30. Michelson D, Malik K, Parikh R, Weiss HA, Doyle AM, Bhat B, et al. Effectiveness of a brief lay counsellordelivered, problem- solving intervention for adolescent mental health problems in urban, low-income schools in India : a randomised controlled trial. Lancet child Adolesc Heal [Internet]. 2020;4642(20). Available from: http://dx.doi.org/10.1016/S2352-4642(20)30173-5

31. Gingerich WJ, Eisengart S. Solution-focused brief therapy: A review of the outcome research. Fam Process. 2000;39(4):477-98.

32. Boyd RC, Lewis J, Borreggine K, Benton TD. Adolescent Depression: Identification and Treatment. Curr Treat Options Pediatr. 2018;4(3):350-62.

33. Hamrin V, Pachler MC. Child \& adolescent depression: review of the latest evidence-based treatments. J Psychosoc Nurs Ment Heal Serv. 2005;43(1):54-63.

34. McCarty CA, Weisz JR. Effects of psychotherapy for depression in children and adolescents: What we can (and can't) learn from meta-analysis and component profiling. J Am Acad Child Adolesc Psychiatry. 2007;46(7):87986.

35. Fréchette-Simard C, Plante I, Bluteau J. Strategies included in cognitive behavioral therapy programs to treat internalized disorders: a systematic review. Cogn Behav Ther [Internet]. 2018;47(4):263-85. Available from: http://doi.org/10.1080/16506073.2017.1388275

36. Verdeli H, Mufson L, Lee L, Keith J. Review of Evidence-Based Psychotherapies for Pediatric Mood and Anxiety 


\section{PROBLEM-SOLVING TRAINING}

Disorders. Curr Psychiatry Rev. 2006;2(3):395-421.

37. Mufson L, Weissman MM, Moreau D, Garfinkel R. Efficacy of interpersonal psychotherapy for depressed adolescents. Arch Gen Psychiatry. 1999;56(6):573-9.

38. Diamond G, Siqueland L. Family Therapy for the treatment of depressed adolescents. Psychotherapy. 1995;32(1):77-90.

39. Hallford DJ, Mellor D. Cognitive-reminiscence therapy and usual care for depression in young adults: Study protocol for a randomized controlled trial. Trials. 2013;14(1):1-8.

40. Richardson ED. Adventure-based therapy and self-efficacy theory: Test of a treatment model for late adolescents with depressive symptomatology. Diss Abstr Int Sect B Sci Eng [Internet]. 2003;63(9-B):4384. Available from: http://ovidsp.ovid.com/ovidweb.cgi?T=JS\&PAGE=reference\&D=psyc4\&NEWS=N\&AN=2003-95006-020

41. Ng MY, Eckshtain D, Weisz JR. Assessing Fit Between Evidence-Based Psychotherapies for Youth Depression and Real-Life Coping in Early Adolescence. J Clin Child Adolesc Psychol. 2016;45(6):732-48.

42. United Nations. Youth [Internet]. 2020 [cited 2020 Nov 4]. Available from: https://www.un.org/en/sections/issues-depth/youth-0/\#: :text=For statistical purposes\%2C however\%2C the,of 15 and 24 years.\&text=This statistically oriented definition of,under the age of 14.

43. Munn Z, Peters MDJ, Stern C, Tufanaru C, McArthur A, Aromataris E. Systematic review or scoping review? Guidance for authors when choosing between a systematic or scoping review approach. BMC Med Res Methodol. 2018;18(1):1-7.

44. Tricco AC, Lillie E, Zarin W, O'Brien KK, Colquhoun H, Levac D, et al. PRISMA extension for scoping reviews (PRISMA-ScR): Checklist and explanation. Ann Intern Med. 2018;169(7):467-73.

45. Cowdell F, Booth A, Appleby B. Knowledge mobilization in bridging patient-practitioner-researcher boundaries: A systematic integrative review protocol. J Adv Nurs. 2017;73(11):2757-64.

46. Whittemore R, Knafl K. The integrative review: Updated methodology. J Adv Nurs. 2005;52(5):546-53.

47. Booth A, Carroll C. Systematic searching for theory to inform systematic reviews: Is it feasible? Is it desirable? Health Info Libr J. 2015;32(3):220-35.

48. Courtney DB, Duda S, Szatmari P, Henderson J, Bennett K. Systematic Review and Quality Appraisal of Practice Guidelines for Self-Harm in Children and Adolescents. Suicide Life-Threatening Behav [Internet]. 2019 Jun 1;49(3):707-23. Available from: https://doi.org/10.1111/sltb.12466

49. Bennett K, Duda S, Brouwers M, Szatmari P, Newton A, McLennan J, et al. Towards high-quality, useful practice guidelines for child and youth mental health disorders: Protocol for a systematic review and consensus exercise. BMJ Open. 2018;8(2).

50. Thomas J, Brunton J, Graziosi S. EPPI-Reviewer 4.0: software for research synthesis. 2010;

51. Peters M, Godfrey C, McInerney P, Munn Z, Trico A, Khalil H. Chapter 11: Scoping Reviews. In: JBI Manual for Evidence Synthesis [Internet]. JBI; 2020. Available from: https://wiki.jbi.global/display/MANUAL/Chapter+11\%3A+Scoping+reviews

52. Sterne JAC, Savović J, Page MJ, Elbers RG, Blencowe NS, Boutron I, et al. RoB 2: A revised tool for assessing risk of bias in randomised trials. BMJ. 2019;366:1-8.

53. Hedges L V. A random effects model for effect sizes. Psychol Bull. 1983;93(2):388-95.

54. Higgins JPT, Thomas J, Chandler J, Cumpston M, Li T, Page MJ, et al. Cochrane Handbook for Systematic Reviews of Interventions. Cochrane Handbook for Systematic Reviews of Interventions. John Wiley \& Sons; 2019.

55. Egger M, Smith GD, Schneider M, Minder C. Bias in meta-analysis detected by a simple, graphical test. Bmj. 1997;315(7109):629-34. 


\section{PROBLEM-SOLVING TRAINING}

56. GRADEpro GDT. GRADEpro Guideline Development Tool [Software] [Internet]. McMaster University (developed by Evidence Prime, Inc.); 2020. Available from: gradepro.org

57. Guyatt GH, Oxman AD, Vist GE, Kunz R, Falck-Ytter Y, Alonso-Coello P, et al. GRADE: an emerging consensus on rating quality of evidence and strength of recommendations. BMJ [Internet]. 2008 Apr 26;336(7650):924-6. Available from: https://www.bmj.com/lookup/doi/10.1136/bmj.39489.470347.AD

58. Heffernan OS, Herzog TM, Schiralli JE, Hawke LD, Chaim G, Henderson JL. Implementation of a youth-adult partnership model in youth mental health systems research: Challenges and successes. Heal Expect. 2017;20(6):1183-8.

59. Hawke LD, Darnay K, Relihan J, Khaleghi-Moghaddam M, Barbic S, Lachance L, et al. Enhancing researcher capacity to engage youth in research: Researchers' engagement experiences, barriers and capacity development priorities. Heal Expect. 2020;23(3):584-92.

60. Hawke LD, Relihan J, Miller J, McCann E, Rong J, Darnay K, et al. Engaging youth in research planning, design and execution: Practical recommendations for researchers. Heal Expect. 2018;21(6):944-9.

61. Becker-Weidman EG, Jacobs RH, Reinecke MA, Silva SG, March JS. Social problem-solving among adolescents treated for depression. Behav Res Ther. 2010 Jan;48(1):11-8.

62. March JS, Silva S, Petrycki S, Curry J, Wells K, Fairbank J, et al. The Treatment for Adolescents with Depression Study (TADS): Long-term effectiveness and safety outcomes. Arch Gen Psychiatry [Internet]. 2007 Oct 1;64(10):1132-44. Available from: http://archpsyc.jamanetwork.com/article.aspx?doi=10.1001/archpsyc.64.10.1132

63. D'Zurilla TJ, Nezu AM, Maydeu- Olivares A. Social Problem-Solving Inventory - Revised: Technical manual. North Tonawanda, NY: Multi-Health Systems; 2002.

64. Kennard BD, Clarke GN, Weersing VR, Asarnow JR, Shamseddeen W, Porta G, et al. Effective Components of TORDIA Cognitive-Behavioral Therapy for Adolescent Depression: Preliminary Findings. J Consult Clin Psychol [Internet]. 2009;77(6):1033-41. Available from: http://doi.apa.org/getdoi.cfm?doi=10.1037/a0017411

65. Brent D, Emslie G, Clarke G, Wagner KD, Asarnow JR, Keller M, et al. Switching to another SSRI or to venlafaxine with or without cognitive behavioral therapy for adolescents with SSRI-resistant depression: The TORDIA randomized controlled trial. JAMA [Internet]. 2008 Feb 27;299(8):901-13. Available from: http://jama.jamanetwork.com/article.aspx?doi=10.1001/jama.299.8.901

66. Brysbaert M. How many participants do we have to include in properly powered experiments? A tutorial of power analysis with some simple guidelines. J Cogn. 2019;2(1):1-38.

67. Dietz LJ, Marshal MP, Burton CM, Bridge JA, Birmaher B, Kolko D, et al. Social problem solving among depressed adolescents is enhanced by structured psychotherapies. J Consult Clin Psychol. 2014;82(2):202-11.

68. Kaufman NK, Rohde P, Seeley JR, Clarke GN, Stice E. Potential mediators of cognitive-behavioral therapy for adolescents with comorbid major depression and conduct disorder. J Consult Clin Psychol. 2005;73(1):38-46.

69. Prinz RJ, Foster S, Kent RN, O'Leary KD. Multivariate assessment of conflict in distressed and nondistressed mother-adolescent dyads. J Appl Behav Anal. 1979;12(4):691-700.

70. World Health Organization. Update of the Mental Health Gap Action Programme (mhGAP) guidelines for mental, neurological and substance use disorders [Internet]. 2015. Available from: https://www.who.int/maternal_child_adolescent/documents/health-promotion-interventions/en/

71. Orygen. Treating depression in young people: Guidance, resources and tools for assessment and management [Internet]. Orygen. 2017. Available from: https://www.orygen.org.au/Training/Resources/Depression/Clinicalpractice-points/Treating-depression-in-yp

72. Ministerio de Salud. Guía Clínica Depresión en personas de 15 años y más [Internet]. Santiago, Chile; 2013. Available from: https://www.minsal.cl/portal/url/item/7222754637c08646e04001011f014e64.pdf

73. Cincinnati Children's Hospital Medical Centre. Best Evidence Statement (BESt): Treatment of children and 


\section{PROBLEM-SOLVING TRAINING}

adolescents with Major Depressive Disorder (MDD) during the Acute Phase. Cincinnati, OH; 2010.

74. Birmaher B. Practice Parameter for the Assessment and Treatment of Children and Adolescents With Depressive Disorders. J Am Acad Child Adolesc Psychiatry. 2007;46(11):1503-26.

75. Wolpert M, Harris R, Hodges S, Fuggle P, James R, Wiener A, et al. THRIVE Framework for system change. London, United Kingdom; 2019.

76. Hostinar CE, Miller GE. Protective factors for youth confronting economic hardship: Current challenges and future avenues in resilience research. Am Psychol. 2019;74(6):641-52.

77. Van Den Heuvel MWH, Bodden DHM, Moerbeek M, Smit F, Engels RCME. Dismantling the relative effectiveness of core components of cognitive behavioural therapy in preventing depression in adolescents: Protocol of a cluster randomized microtrial. BMC Psychiatry. 2019;19(1):1-13.

78. Nezu AM, Perri MG. Social Problem-Solving Therapy for Unipolar Depression: An Initial Dismantling Investigation. J Consult Clin Psychol. 1989;57(3):408-13.

79. D'Zurilla TJ, Nezu AM. Problem-solving therapy: a positive approach to clinical intervention. 3rd ed. New York, NY: Spring Publishing Company; 2007. 261 p.

80. Chang EC, D'Zurilla TJ, Sanna LJ. Social problem solving: Theory, research, and training. American Psychological Association; 2004.

81. Nezu AM, Nezu CM, Perri MG. Problem-Solving Therapy for Depression: Theory, Research, and Clinical Guidelines. New York, NY: Wiley; 1989.

82. Nezu AM. A problem-solving formulation of depression: A literature review and proposal of a pluralistic model. Clin Psychol Rev. 1987;7(2):121-44.

83. Goodman R, Meltzer H, Bailey V. The Strengths and Difficulties Questionnaire: a pilot study on the validity of the self-report version. Eur Child Adolesc Psychiatry. 1998 Sep;7(3):125-30.

84. Goodman R. The Strengths and Difficulties Questionnaire: A Research Note. J Child Psychol Psychiat. 1997;38(5):581-6.

85. Beck AT, Steer RA. Internal consistencies of the original and revised beck depression inventory. J Clin Psychol. 1984;40(6):1365-7.

86. Radloff LS. The CES-D Scale: A Self-Report Depression Scale for Research in the General Population. Appl Psychol Meas [Internet]. 1977 Jun 26;1(3):385-401. Available from: http://journals.sagepub.com/doi/10.1177/014662167700100306

87. Goodman R. Psychometric properties of the Strengths and Difficulties Questionnaire (SDQ). J Am Acad Child Adolesc Psychiatry. 2001;40:1337-45.

88. Beck AT, Steer RA, Brown GK. Beck Depression Inventory-ii. San Antonio, TX: Psychological Corporation; 1996.

89. Poznanski E, Mokros H. Children's Depression Rating Scale-Revised (CDRS-R). Los Angeles, CA: Western Psychological Services; 1996.

90. Busner J, Targum SD. The clinical global impressions scale: applying a research tool in clinical practice. Psychiatry (Egmont). 2007;4(7):28-37.

91. Kaufman J, Birmaher B, Brent D, Rao U, Flynn C, Moreci P, et al. Schedule for affective disorders and schizophrenia for school-age children-present and lifetime version (K-SADS-PL): Initial reliability and validity data. J Am Acad Child Adolesc Psychiatry [Internet]. 1997 Jul;36(7):980-8. Available from: http://linkinghub.elsevier.com/retrieve/pii/S0890856709625557 